\section{Visualizing Gene Expression}

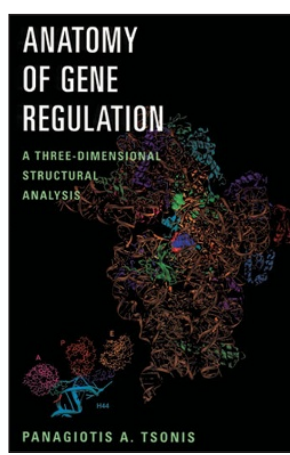

\section{Anatomy of Gene Regulation: A Three-dimensional Structural Analysis}

by Pangiotis A. Tsonis

Cambridge University Press, \$50/\$140

296 pp. paperback/hardback

ISBN 0521800307, January 2003

Reviewed by Stephen Buratowski

This year we celebrate the impact of Watson and Crick's DNA structure on the subsequent 50 years of molecular biology. This is undoubtedly the most famous of many examples in which structural studies have illuminated a biological process. In the past few years, we've seen beautiful and informative three-dimensional images of the nucleosome, RNA and DNA polymerases and the ribosome. Years of genetic and biochemical data spring to life when mapped onto these structures, and future experiments can be designed with much greater precision. Many of us who teach molecular biology at the undergraduate or graduate level have tried to convey this excitement by incorporating structures into our teaching. What might otherwise be dry descriptions of protein interactions or catalytic steps take on greater clarity and tangibility when students can see the molecules.

With this goal in mind, Pangiotis Tsonis has compiled and summarized a great deal of structural information in his new book entitled Anatomy of Gene Regulation: A Three-Dimensional Structural Analysis. The book is not meant to be a comprehensive textbook. Instead, it is meant to supplement the more schematic descriptions of the central dogma found in standard texts by supplying pictures and insight gleaned from X-ray crystallography, NMR spectroscopy and highresolution microscopy. It's a terrific idea and a laudable effort, but several problems prevent the book from fulfilling its promise.

Stephen Buratowski is in the Department of Biological Chemistry \& Molecular Pharmacology, Harvard Medical School, Boston, Massachusetts.
The chapters of the book are nicely organized around individual steps in gene expression: replication, transcription, translation, and so on. The target audience appears to be advanced undergraduates and graduate students. The text describes key features of the various structures, but because this is not a comprehensive textbook, the details will only make sense to those already familiar with the processes. For those interested in a deeper understanding of the structures, the brief summaries in the book will be much less useful than the original articles.

Several things detract from the presentation of the structures. Almost all the figures are taken directly from the original journal articles. It's an interesting chronicle of the improvements in computer graphics over the last decade, but makes for an inconsistency that doesn't serve the reader well. Every figure has a different color scheme and a different way to represent proteins and nucleic acids. Since the original figure legends are not included, some features in the figures are not clearly described in the text. For a book built around visualization of molecules, the figures specifically made for the book are of surprisingly poor quality. They appear to be low-resolution screen shots from a computer monitor. Another problem is that the original journal articles are cited by the name of the senior author rather than the first author. This practice is disrespectful of the postdocs and graduate students that did the work and makes it almost impossible to match the citations to the references listed at the end of the book.

While many of these problems can be remedied in future editions of the book, a more fundamental issue is raised. When the goal is to convey three-dimensional information about molecules, is a static twodimensional medium like print the appropriate vehicle? Now that molecular structures can be viewed and manipulated with simple programs running on personal computers, I would argue that the time has come to move beyond a simple textbook. A well-designed online site, allowing students to see the important features of molecules from all angles and at various resolutions, would be a more powerful teaching tool. Such a resource could either stand alone or be combined with a textbook. Some of the basic molecular biology textbooks now use auxiliary websites or CD-ROMs to present structures, and a similar online extension to Anatomy of Gene Regulation would greatly increase its teaching utility. Structural biology will undoubtedly continue its rapid progress and these findings will increasingly become part of the undergraduate and graduate curricula. Efforts such as this book are taking us in the right direction, but it's time to make use of the newer technologies available to us. 